\title{
Prospective comparison of clinical and echocardiographic diagnosis of rheumatic carditis: long term follow up of patients with subclinical disease
}

\author{
F E Figueroa, M Soledad Fernández, P Valdés, C Wilson, F Lanas, F Carrión, X Berríos, \\ $F$ Valdés
}

\begin{abstract}
Objective-To determine the frequency of occurrence and long term evolution of subclinical carditis in patients with acute rheumatic fever.

Design-Valvar incompetence was detected by clinical examination and Doppler echocardiographic imaging during the acute and quiescent phases of rheumatic fever. Patients were followed prospectively and submitted to repeat examinations at one and five years after the acute attack. Persistence of acute mitral and aortic lesions detected solely by echocardiography (subclinical disease) was compared with that of disease detected by clinical examination as well (thereby fulfilling the latest 1992 Jones criteria for rheumatic carditis).

Setting-Three general hospitals with a university affiliation in Chile.

Patients-35 consecutive patients fulfilling the revised Jones criteria for rheumatic fever. Clinical and echocardiographic examination was repeated in 32 patients after one year and in 17 after five years. Ten patients had subclinical carditis on admission, six of whom were followed for five years.

Main outcome measures-Auscultatory and echocardiographic evidence of mitral or aortic regurgitation during the acute attack or at follow up.

Results-Mitral or aortic regurgitation was detected by Doppler echocardiographic imaging in $25 / 35$ rheumatic fever patients as opposed to $5 / 35$ by clinical examination $(p=0.03)$. Doppler echocardiography revealed acute valvar lesions in 10 of 20 rheumatic fever patients who had no auscultatory evidence of rheumatic carditis (subclinical carditis). Three of these subclinical lesions and three of the clinical or auscultatory lesions detected on admission were still present after five years of follow up, emphasising that subclinical lesions are not necessarily transient.

Conclusions-Doppler echocardiographic imaging improves the detection of rheumatic carditis. Subclinical valve lesions, detected only by Doppler imaging, can persist. Echocardiographic findings should be accepted as a major criterion for the diagnosis of rheumatic fever.

(Heart 2001;85:407-410)
\end{abstract}

Keywords: rheumatic heart disease; rheumatic fever; echocardiography; carditis

Acute rheumatic fever and rheumatic heart disease continue to be a major health problem in developing countries, and rheumatic fever is the leading cause of acquired heart disease in children and young adults worldwide. ${ }^{12}$ With the recent advent of cross sectional echocardiography and colour flow Doppler imaging, it has been claimed that mitral and aortic valve insufficiency can be detected in up to $90 \%$ of rheumatic fever patients who have no clinical evidence of carditis. ${ }^{3}$ Nonetheless, the 1992 update of the Jones criteria excludes the use of echocardiography, including Doppler, to document valve disease in the absence of auscultatory signs of carditis. ${ }^{5}$ One concern has been the finding of silent mitral regurgitation in normal subjects, which may be a cause of overdiagnosis of rheumatic fever. ${ }^{6}$ Several investigators have addressed this point, ${ }^{37}$ and two groups have carried out blinded tests of their ability to distinguish physiological from pathological patterns of regurgitation in rheumatic fever patients and controls. ${ }^{48}$

A remaining issue relating to colour flow Doppler echocardiography in rheumatic fever has been uncertainty over the long term significance of abnormal regurgitant flow patterns when carditis is not clinically apparent. ${ }^{39}$ Few studies have addressed this problem prospectively, and follow up has been of limited duration. $^{3} 1011$ We therefore conducted a prospective multicentre clinical and Doppler echocardiographic survey of a cohort of 35 rheumatic fever patients fulfilling the Jones diagnostic criteria. ${ }^{12}$ We now report our findings, including those cases with clinically silent (subclinical) rheumatic carditis, who were followed with repeat examinations over a period of five years.

\section{Methods}

From February 1992 to December 1993 we enrolled prospectively 35 consecutive patients fulfilling the revised Jones criteria for rheumatic fever. ${ }^{12}$ These where admitted to the Catholic University hospital in Santiago ( $n=4)$, the Sótero del Río hospital in the south east metropolitan area of Santiago $(n=17)$, or the regional hospital of Temuco in Chile's ninth region $(n=14)$. 
Table 1 Baseline characteristics and major fones criteria of patients with acute rheumatic fever on entry to the study

\begin{tabular}{ll}
\hline Total number of ARF patients & 35 \\
\hline Sex & \\
$\quad$ Male & $21(60)$ \\
$\quad$ Female (me (years) (mean (range)) & $14(40)$ \\
First ARF episode & $15(5-34)$ \\
Polyarthritis & $29(83)$ \\
Carditis & $28(80)$ \\
Chorea & $15(43)$ \\
Erythema marginatum & $4(11)$ \\
Subcutaneous nodules & $1(3)$ \\
\end{tabular}

Values are $\mathrm{n}$ or $\mathrm{n}(\%)$ unless stated otherwise.

Raised anti-streptolysin $\mathrm{O}$ and/or anti-DNAse-B titres were present in all patients.

ARF, acute rheumatic fever.

On entry, patients underwent a standardised examination protocol consisting of a detailed medical history recorded by a physician, and general and specific laboratory tests, including an ECG, a chest $x$ ray, throat swab cultures, and quantification of anti-streptolysin $\mathrm{O}$ (ASO) and anti-DNAse-B (ADB). All patients were examined on repeated occasions throughout the investigation by one or two of the investigators (MF, CW, or FL-all cardiologists experienced in the care of patients with rheumatic fever). These investigators both completed the standardised protocol and performed the Doppler echocardiography studies, thus enabling the additional information provided by echocardiography to be evaluated in clinical practice.

As our main purpose was to compare the long term outcome of clinical and subclinical valvar lesions, blinding of clinicians to the echocardiographic studies was not considered necessary. Disease manifestations and auscultatory findings were evaluated according to criteria published by the World Health Organization $^{13}$ and by Taranta and Markowitz, ${ }^{14}$ as described elsewhere. ${ }^{15}$ Cross sectional echocardiography and colour Doppler evaluation was performed within five days of admission, on discharge, and at three months, 12 months, and five years from entry to the study. We initially employed an Aloka SSD-870 (Aloka Co Ltd, Mure, Mitaki-shi, Tokyo, Japan) and then a Hewlett-Packard 1000 or 1500 ultrasound system, with 2.7 and $3.5 \mathrm{MHz}$ transducers for pulsed and continuous wave Doppler and colour flow imaging (Hewlett-Packard Inc, Andover, Massachusetts, USA). A $1.9 \mathrm{MHz}$ imageless pencil-type probe was also employed for

Table 2 Clinical (auscultatory) and echo Doppler detection of valve lesions in patients with acute rheumatic fever during the acute episode and during follow up

\begin{tabular}{|c|c|c|c|c|c|c|}
\hline \multirow[b]{3}{*}{ Valve involvement } & & & \multicolumn{4}{|l|}{ Follow up } \\
\hline & \multicolumn{2}{|c|}{ ARF at entry $(n=35)$} & \multicolumn{2}{|c|}{1 year $(n=32)$} & \multicolumn{2}{|c|}{5 years $(n=17)$} \\
\hline & Clinical & $2 D$-echo- $D$ & Clinical & $2 D$-echo- $D$ & Clinical & $2 D$-echo-D \\
\hline Mitral & $15(42 \%)$ & $20(75 \%)$ & $7(22 \%)$ & $12(32 \%)$ & $3(18 \%)$ & $5(29 \%)$ \\
\hline Aortic & $6(17 \%)$ & $17(48 \%)^{\star}$ & $2(6 \%)$ & $9(28 \%) \dagger$ & $0(0 \%)$ & $3(18 \%)$ \\
\hline Mitral and/or aortic & $5(43 \%)$ & $25(71 \%)^{\star \star}$ & $8(25 \%)$ & $15(47 \%)$ & $3(18 \%)$ & $7(41 \%)$ \\
\hline Not detected & $20(57 \%)$ & $10(29 \%)$ & $24(75 \%)$ & $17(53 \%)$ & $14(82 \%)$ & $10(59 \%)$ \\
\hline
\end{tabular}

www.heartjinl.com continuous Doppler. Multiple cross sectional views were taken from parasternal, apical, and subcostal positions according to the recommendations of the American Society of Echocardiography. ${ }^{16}$

Criteria for pathological mitral regurgitation, previously agreed upon by the authors, were as follows:

- colour jet identified in at least two planes;

- mosaic colour jet;

- persistence of the jet throughout systole.

The length of the colour jet was not necessarily $>1 \mathrm{~cm}$ in all cases. Clinical, echocardiographic, and laboratory data on the evolution of each episode were collected in specially designed computerised data collection forms.

One patient refused follow up after the first week and one was lost after three months. An additional patient was excluded from final analysis because he required valve surgery after 10 months. The remaining 32 patients are included in our one year follow up report. Seventeen of these patients were evaluated again at five years after entry to the study, with an average follow up of five years and seven months.

\section{STATISTICS}

Data from the groups were compared by $t$ test. When data were not normally distributed, the non-parametric Mann-Whitney U test, the $\chi^{2}$ test, and the Fisher exact test were used. Probability values of $p<0.05$ were considered significant.

\section{Results}

The baseline features of this group of 35 rheumatic fever patients are shown in table 1 . Fifteen patients $(43 \%)$ had clinical evidence of carditis according to classical criteria. ${ }^{14}{ }^{15}$ Fulfilment of the revised Jones criteria ${ }^{12}$ was a prerequisite for enrolment, but in retrospect patients also satisfied the 1992 updated Jones criteria. $^{5}$

A comparison of clinical and echocardiographic diagnosis of rheumatic carditis is given in table 2. During the rheumatic fever episode and also during follow up, Doppler echocardiography detected more valvar lesions than clinical examination. This difference was significant for all lesions (mitral or aortic) during the acute episode $(p=0.03)$ and for aortic lesions, both during the acute attack $(p=0.01)$ and one year later $(p=0.025)$. Doppler echocardiography detected aortic regurgitation in 11 more patients $(31 \%)$ than clinical examination, and it detected mitral regurgitation in five more patients $(15 \%)$ than clinical examination.

At entry, 25 patients had echocardiographic evidence of valvar (mitral or aortic) incompetence (table 2). Among these cases, we found 10 individuals with no auscultatory or clinical findings suggesting acute rheumatic carditis, in spite of repeated examinations (fig 1). These 10 individuals constitute our group of rheumatic fever patients with subclinical carditis. They represent almost $30 \%$ (10 of 35) of all the rheumatic fever patients entering the study (fig 1). Purely echocardiographic evidence of acute rheumatic carditis (that is, subclinical carditis) 

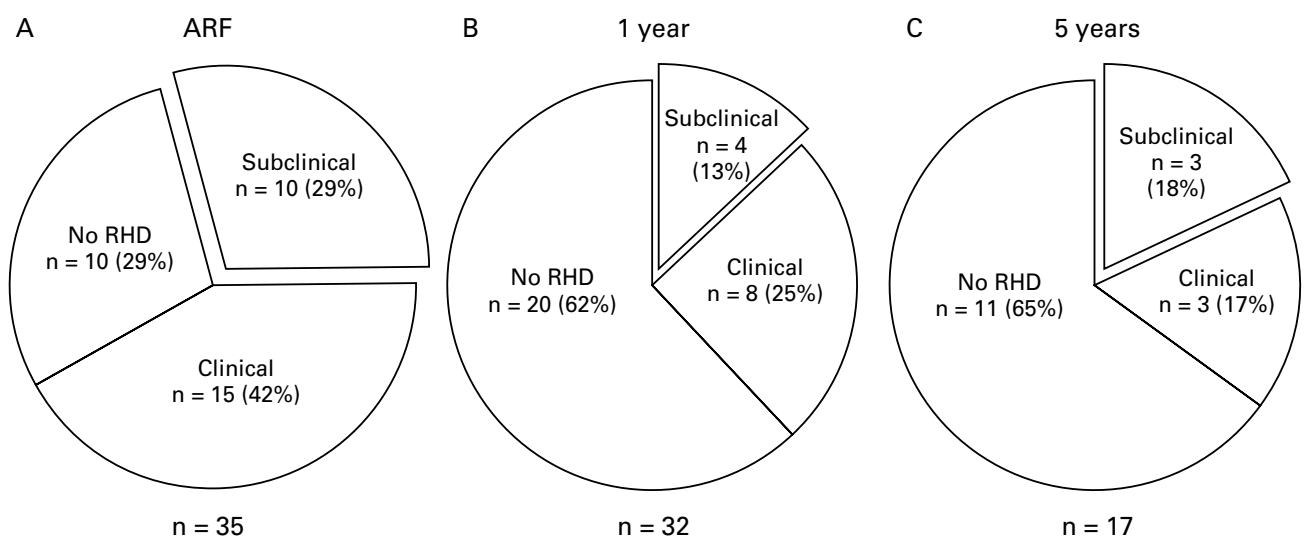

Figure 1 Subclinical and clinical valve lesions in rheumatic fever patients at entry $(A)$ and at one year $(B)$ and five years (C) follow up. ARF, acute rheumatic fever; No RHD, no clinical or echocardiographic evidence of rheumatic heart disease.

was found in 10 of the 20 rheumatic fever patients who had no clinical signs or auscultatory evidence of acute rheumatic carditis at entry (table 2 and fig 1 ). Five of these cases had aortic regurgitation, four had mitral insufficiency, and one had mitral-aortic disease. This last case was classified as moderate, ${ }^{14}$ while all the other cases were considered mild. None had annular dilatation, elongation of chordae to the anterior leaflet, valve prolapse, thickening, focal nodularities, or limitation of aperture. Nine of the patients had polyarthritis and one had Sydenham's chorea as major Jones criteria. Six had systolic murmurs of typically innocent quality; such murmurs were also found in a similar proportion in the group without carditis.

As the high sensitivity of ultrasound in detecting mitral and aortic regurgitation is well known, ${ }^{34}$ our main objective was rather to assess the long term significance of subclinical valve disease in rheumatic fever. We therefore sought to compare how often the finding of a clinical or a subclinical valve lesion, detected at entry to the study, would persist after one or five years of follow up.

Figure 1 shows the percentage of rheumatic fever patients who were found to have subclinical valve disease (subclinical), clinically evident or auscultatory disease (clinical), or no detectable valve lesions, either clinical or echocardiographic (no rheumatic heart disease) during the acute episode (fig 1A) and after one year (fig 1B) and five years (fig 1C) of follow up. As expected, in the group of 32 rheumatic fever cases with repeat examination at one year and in the 17 cases re-examined at five years, the percentage of individuals with subclinical valve lesions, as well as those with clinical lesions, decreased with time. Nonetheless, some subclinical valve lesions were found to persist after five years of follow up ( $3 / 17 ; 18 \%)$ (fig $1 \mathrm{C}$ ). In fact, as shown in table 3 , half (three of six) of our cases of subclinical carditis who were followed for five years still had valvar incompetence, detected only by Doppler echocardiography, at the end of the study. Furthermore, when we compared the percentage of clinical or subclinical valve lesions that persisted throughout follow up, we found no significant differences after one year $(57 \% v 44 \%)$ or five years
Table 3 Persistence of valvar lesions in patients who had subclinical (only echocardiographic) or clinical

(auscultatory) carditis at entry, when re-examined one and five years after the episode of acute rheumatic fever ( $A R F)$

\begin{tabular}{lll}
\hline \multirow{2}{*}{$\begin{array}{l}\text { Valve involvement during ARF } \\
\text { episode }\end{array}$} & \multicolumn{2}{l}{ Persisting lesions } \\
\cline { 2 - 3 } & After 1 year & After 5 years \\
\hline Clinical $(\mathrm{n}=15)$ & $8 / 14$ & $3 / 5$ \\
Subclinical $(\mathrm{n}=10)$ & $4 / 9$ & $3 / 6$
\end{tabular}

The percentage of clinical and subclinical valve lesions still detectable after follow up was not different after one year $(p=$ $0.68)$ or five years $(p=1.0)$.

$(60 \%$ v 50\%) (table 3), suggesting that acute valve lesions induced by subclinical rheumatic carditis persist to about the same extent as the lesions in clinically evident acute rheumatic heart disease.

No individual with subclinical carditis developed a clinically detectable or auscultatory lesion during follow up; however, one case was of special interest as his subclinical mitralaortic disease initially resolved during follow up but reappeared again after five years, with no evidence of recurrence of rheumatic fever.

\section{Discussion}

Clinically manifest mitral or aortic regurgitation is still considered the diagnostic hallmark of acute rheumatic carditis. ${ }^{67}$ However, our findings confirm previous reports that Doppler echocardiography can detect significant valvar incompetence in the absence of auscultatory findings, during both the acute and the quiescent phases of the disease. ${ }^{34717} 18$ In the well known recent outbreak of rheumatic fever in the USA, carditis was diagnosed by auscultation in 53 of the 74 patients $(72 \%)$, while Doppler echocardiography detected mitral regurgitation in an additional 14 patients $(19 \%) .{ }^{19}$ The same group also detected subclinical carditis in $47 \%$ of rheumatic fever patients presenting with polyarthritis and $57 \%$ of patients with "pure" chorea, ${ }^{7}$ as we did in $50 \%$ of our own cases with no clinical evidence of rheumatic heart disease. Similar findings have been reported by Folger and colleagues in the Middle East ${ }^{3}$ and by Abernethy and associates in New Zealand. ${ }^{4}$ In our series, roughly one third of rheumatic fever patients had subclinical carditis, in spite of being repeatedly 
examined by cardiologists experienced in rheumatic fever. Several reports suggest that active rheumatic carditis may be clinically silent or unsuspected; for example, $30-40 \%$ of adults with mitral stenosis cannot recall an illness suggesting rheumatic fever. ${ }^{20}$ On the other hand, studies of patients with isolated chorea suggest that subclinical rheumatic heart disease may lead to irreversible sequelae. Carapetis and Currie recently reported that the majority $(68 \%)$ of people with chorea who develop chronic rheumatic heart disease have no evidence of carditis at the time of their initial attack of rheumatic fever. ${ }^{21}$ Also, follow up of patients with isolated chorea for 20 years showed that $23 \%$ developed mitral stenosis. ${ }^{22}$

Only one prospective study from India has failed to find evidence of Doppler regurgitation in rheumatic fever patients without clinical evidence of carditis. ${ }^{10}$ This discrepancy has been attributed to the presumption that in developing countries patients may seek medical attention only in a late phase of the disease, when clinical valvar involvement is evident. This would be concordant with the findings from a study in New Zealand, where all patients with subclinical carditis developed an audible murmur within the next two weeks. ${ }^{4}$ In contrast, none of our patients with only Doppler evidence of carditis developed new murmurs during follow up. This does not imply that the finding of subclinical carditis is irrelevant. Veasy and colleagues reported a case of infective endocarditis in a patient with subclinical carditis, ${ }^{7}$ and Folger and associates found that four of six patients with only Doppler evidence of valve involvement continued to show valvar regurgitation $18-36$ months later. ${ }^{3}$ In one or our cases, subclinical valvar disease became evident again, after initial resolution, with no known recurrence of rheumatic fever.

Except for the data from our present report, there is no available information on the long term evolution of subclinical carditis in rheumatic fever patients. ${ }^{23}$ In this regard, our most important observation is that clinically silent carditis is not necessarily a benign or transient entity, because at least in $60 \%$ of cases, valvar disease persisted after five years of follow up in spite of continuous penicillin prophylaxis and no evidence of recurrent disease. As seen in table 3, the persistence of subclinical valve lesions was similar to that found in the cases with clinical or auscultatory carditis. As all our patients fulfilled the Jones diagnostic criteria as a prerequisite for entry to the study and no case of subclinical carditis was severe, the contribution of Doppler echocardiography in these cases did not alter the management of the episode of rheumatic fever. However, these findings could be important with respect to long term prognosis and the development of late cardiovascular sequelae, and therefore may be relevant to decisions regarding penicillin prophylaxis.
CONCLUSIONS

These findings confirm that Doppler echocardiography improves the detection of rheumatic carditis and chronic rheumatic heart disease and show that subclinical valve lesions can persist. They lend support to the view that echocardiographic findings should be accepted as a major criterion for the diagnosis of rheumatic fever. ${ }^{24}$

This work was funded by grant No 1960117 from Fondo Nacional de Desarrollo Científico y Tecnológico (Fondecyt), Chile. The study was presented at the annual meeting of the Chile. The study was presented at the annual meeting of the
American College of Rheumatology, Boston, Massachusetts, American College of R
November 13-17, 1999.

1 Nordet P. WHO/ISFC Global programme for the prevention and control of RF/RHD. F Int Fed Cardiol 1993;3:4-5.

2 Eisenberg MJ. Rheumatic heart disease in the developing world: prevalence, prevention and control. Eur Heart $\mathfrak{f}$ world: prevalence,

3 Folger GM, Hajar R, Robida A, et al. Occurrence of valvar heart disease in acute rheumatic fever without evident carditis: colour flow Doppler identification. Br Heart $\mathcal{f}$ 1992;67:434-8

4 Abernethy M, Bass N, Sharpe N, et al. Doppler echocardiography and the early diagnosis of carditis in acute rheumatic fever. Aust N Z Med 1994;24:530-5.

5 Special Writing Group of the Committee on Rheumatic Fever, Endocarditis and Kawasaki Disease of the Council on Cardiovascular Disease in the Young of the American Heart Association. Guidelines for the diagnosis of rheumatic fever. Jones criteria, 1992 update. $7 A M A$ 1992;268: 2069-73.

6 Dajani AS, Allen HD, Taubert KA. Echocardiography for diagnosis and management of rheumatic fever [letter]. diagnosis and manag

7 Veasy LG, Tani LY, Hill HR. Persistence of acute rheumatic fever in the intermountain area of the United States. $\mathcal{F}$ Pediatr 1994;124:9-16.

8 Minich LL, Tani LY, Pagotto LT, et al. Doppler echocardiography distinguishes between physiologic and pathologic "silent" mitral regurgitation in patients with rheumatic fever. Clin Cardiol 1997;20:924-6.

9 Stollerman GH. Seminar: rheumatic fever. Lancet 1997;349: 935-42.

10 Vasan R, Shrivastava S, Vijiyakumar M, et al. Echocardiographic evaluation of patients with acute rheumatic fever and rheumatic carditis. Circulation 1996;94:73-82.

11 Hilário MO, Gasparian AB, Carvalho AC, et al. Echocardiographic findings in patients with acute rheumatic fever (ARF) without clinical evidence of carditis-a prospective (ARF) without clinical evidence of carditis-a prospective
blind study [abstract]. Arthritis Rheum 1997;40(suppl): S285.

12 American Heart Association. Jones criteria (revised) for guidance in the diagnosis of rheumatic fever. Circulation 1984;69:204-8A.

13 World Health Organization Study Group. Rheumatic fever and rheumatic heart disease. (Technical Report Series No 764.) Geneva: World Health Organization, 1988.

14 Taranta A, Markowitz M: Rheumatic fever, 2nd ed. Boston, Mass, Kluwer Academic Publishers, 1989.

15 Figueroa F, Berríos X, Gutierrez M, et al. Anticardiolipin antibodies in acute rheumatic fever. $\mathcal{F}$ Rheumatol 1992;19: 1175-80.

16 Feigenbaum H. Echocardiography, 4th ed. Philadelphia: Lea and Febiger, 1986:127-87.

17 Veasy LG. Echocardiography for diagnosis and management of rheumatic fever. ҰAMA 1993;269:2084.

18 Wilson NJ, Neutze JM. Echocardiographic diagnosis of subclinical carditis in acute rheumatic fever. Int $\mathcal{F}$ Cardiol 1995;50:1-6.

19 Veasy LG, Wiedmeier SE, Orsmond GS, et al. Resurgence of acute rheumatic fever in the intermountain area of the United States. N Engl F Med 1987;316:421-7.

20 Bland EF, Jones TD. Rheumatic fever and rheumatic heart disease. A twenty year report on 1000 patients followed since childhood. Circulation 1951;4:836-43.

21 Carapetis JR, Currie BJ. Rheumatic chorea in northern Australia: a clinical and epidemiological study. Arch Dis Child 1999;80:353-8.

22 Bland EF. Chorea as a manifestation of rheumatic fever. A long term perspective. Trans Am Clin Climatol Assoc 1961;73:209-13.

23 Narula J, Chandrasekhar Y, Rahimtoola S. Diagnosis of active rheumatic carditis. The echoes of change. Circulation 1999;100:1576-81.

24 Wilson NJ, Neutze JM. Echocardiographic diagnosis of mitral insufficiency [letter]. F Pediatr 1994;125:673. 